Pacific Journal of Mathematics

CODIMENSION TWO ISOMETRIC IMMERSIONS BETWEEN 


\title{
CODIMENSION TWO ISOMETRIC IMMERSIONS BETWEEN EUCLIDEAN SPACES
}

\author{
LEE WHITT
}

\begin{abstract}
Hartman and Nirenberg showed that any $C^{\infty}$ isometric immersion $f$ : $\mathbf{E}^{n} \rightarrow \mathbf{E}^{n+1}$ between flat Euclidean spaces is a cylinder erected over a plane curve. We show that in the codimension two case, $f: \mathbf{E}^{n} \rightarrow \mathbf{E}^{n+2}$ factors as a composition of isometric immersions $f=f_{1} \circ f_{2}: \mathbf{E}^{n} \rightarrow \mathbf{E}^{n+1}$ $\rightarrow \mathbf{E}^{n+2}$, when $n>1$ and $f$ has nowhere zero normal curvature. Counterexamples are given if this assumption is relaxed.
\end{abstract}

How can paper be folded? More precisely, how can flat Euclidean 2-space $\mathbf{E}^{2}$ be isometrically immersed into flat Euclidean $n$-space $\mathbf{E}^{n}$ (for simplicity, assume $C^{\infty}$ differentiability everywhere). For $n=3, \mathrm{~A}$. V. Pogorelov [4] announced without proof that the image is a cylinder erected over a plane curve; proofs may be found in Massey [3] and Stoker [5]. In this paper, we consider $n=4$ and show that any isometric immersion $g: \mathbf{E}^{2} \rightarrow \mathbf{E}^{4}$ with nowhere zero normal curvature factors as a composition of isometric immersions $g=g_{1} \circ g_{2}: \mathbf{E}^{2} \rightarrow \mathbf{E}^{2} \rightarrow \mathbf{E}^{4}$.

The result of Pogorelov has been generalized by Hartman and Nirenberg [2]. They showed that the image of any codimension-one isometric immersion between flat Euclidean spaces is a cylinder erected over a plane curve. Using a result of Hartman [1] we easily show that any codimensiontwo, isometric immersion $f: \mathbf{E}^{n} \rightarrow \mathbf{E}^{n+2}, n>1$, with nowhere zero normal curvature factors as a composition $f=f_{1} \circ f_{2}: \mathbf{E}^{n} \rightarrow \mathbf{E}^{n+1} \rightarrow \mathbf{E}^{n+2}$. The images of $f_{1}$ and $f_{2}$ are cylinders. The assumption of nowhere zero normal curvature is essential; counterexamples are given in $\$ 3$ when the assumption is relaxed.

From another point of view, the cylinders of Pogorelov and Hartman and Nirenberg can be deformed ("unrolled") through a one-parameter family of isometric immersions to a hyperplane. This family is obtained by deforming the generating plane curve to a straight line. From our results, it follows easily that any isometric immersion $f: \mathbf{E}^{n} \rightarrow \mathbf{E}^{n+2}$ with nowhere zero normal curvature can be deformed through isometric immersions to a standard inclusion $i: \mathbf{E}^{n} \hookrightarrow \mathbf{E}^{n+2}$ (it would be interesting to know if the normal curvature assumption can be removed). In addition, we proved [7] that if the normal curvature is identically zero, then any 
isometric immersion $h: \mathbf{E}^{n} \rightarrow \mathbf{E}^{m}$ (any codimension) is deformable through isometric immersions to a standard inclusion. In codimension one, the normal curvature is always zero.

We thank J. D. Moore for bringing our attention to [1].

1. Preliminaries. We begin with some Riemannian geometry. As mentioned earlier, $C^{\infty}$ differentiability is assumed everywhere.

Let $h: M \rightarrow \bar{M}$ be an isometric immersion between Riemannian manifolds. Let $\nabla, \bar{\nabla}$ be the Riemannian connection on $M, \bar{M}$ respectively, and let $T M$ and $\nu(h)$ denote the tangent and normal bundles. If $X \in \Gamma(T M)$ and $N \in \Gamma(\nu(h))$ are sections of the tangent and normal bundles, then we can decompose $\bar{\nabla}_{X} N$ into its tangential and normal components $\bar{\nabla}_{X} N=A_{X} N+D_{X} N$. The linear mapping $A_{p}: T_{p} \otimes \nu_{p}(h) \rightarrow$ $T_{p} M$ is the second fundamental form of $h$ at $p \in M$, and $D$ is the normal connection. It is easy to see that $\nu(h)$ is a Riemannian vector bundle with the induced metric and Riemannian connection $D$. We will use $\langle$,$\rangle to$ denote the metric on both $\nu(h)$ and TM. Associated to $A$ is the second fundamental tensor $B: T M \otimes T M \rightarrow \nu(h)$ defined by $\langle B(X, Y), N\rangle=$ $\left\langle A_{N} X, Y\right\rangle$. The curvature tensors associated to $\nabla$ and $D$ are given by

$$
\begin{aligned}
& R(X, Y) Z=\nabla_{X} \nabla_{Y} Z-\nabla_{Y} \nabla_{X} Z-\nabla_{[X, Y]} Z \\
& R^{*}(X, Y) N=D_{X} D_{Y} N-D_{Y} D_{X} N-D_{[X, Y]} N
\end{aligned}
$$

where $X, Y, Z$ are tangent vector fields on $M$, and $N$ is a normal vector field on $M$.

Now we specialize to $\bar{M}=\mathbf{E}^{m}$. The following equations are necessary and sufficiently conditions for the existence of $h: M \rightarrow \mathbf{E}^{m}$ (see [6]).

$$
\begin{gathered}
R(X, Y) Z=A_{X} B(Y, Z)-A_{Y} B(X, Z) \\
R^{*}(X, Y) N=B\left(A_{X} N, Y\right)-B\left(X, A_{Y} N\right) \\
\begin{array}{c}
\nabla_{X} A_{Y} N-\nabla_{Y} A_{X} N-A_{[X, Y]} N \\
=A_{Y} D_{X} N-A_{X} D_{Y} N
\end{array}
\end{gathered}
$$

(Codazzi-Mainardi).

EXISTENCE TheOREM. Let $M$ be a simply connected Riemannian $n$ manifold with a Riemannian $k$-plane bundle $\nu$ over $M$ equipped with a second fundamental form $A$, an associated second fundamental tensor $B$, and $a$ compatible normal connection $D$ (compatible with the Riemannian metric on $\nu$ ). If the Gauss and Codazzi-Mainardi equations are satisfied, then $M$ can be isometrically immersed in $\mathbf{E}^{n+k}$ with normal bundle, $\nu$, normal connection $D$, and second fundamental form $A$. 
The rigidity theorem states that an isometric immersion is essentially determined by its Riemannian data.

Rigidity THEOREM. Let $h, h^{\prime}: M \rightarrow \mathbf{E}^{n+k}$ be isometric immersions of a connected Riemannian n-manifold (not necessarily simply connected) with normal bundles $\nu, \nu^{\prime}$ equipped as above with bundle metrics, connections, and second fundamental forms. Suppose that there is an isometry $\phi: M \rightarrow M$ that can be covered by a bundle map $\phi^{*}: \nu \rightarrow \nu^{\prime}$ which preserves the bundle metrics, the connections, and the second fundamental forms. Then there is an isometry $\varphi$ of $\mathbf{E}^{n+k}$ such that $\varphi \circ h=h^{\prime} \circ \phi$.

In [7], a one parameter version of the existence theorem is established. This gives the deformations discussed in the introduction.

2. The results. We will prove,

THEOREM 1. Let $f: \mathbf{E}^{n} \rightarrow \mathbf{E}^{n+2}, n>1$, be an isometric immersion with nowhere zero normal curvature. Then $f$ factors as the composition of isometric immersions $f=f_{1} \circ f_{2}: \mathbf{E}^{n} \rightarrow \mathbf{E}^{n+1} \rightarrow \mathbf{E}^{n+2}$.

COROLlaRY 1. There is a deformation through isometric immersions between $f$ and the standard inclusion $i: \mathbf{E}^{n} \rightarrow \mathbf{E}^{n+2}$.

Proof. The deformation is obtained by first unrolling $f_{1}$ and then $f_{2}$.

Q.E.D.

The proof of the theorem consists of setting up and solving the associated algebraic problem at the bundle level, and then apply the existence theorem to obtain the required isometric immersions. The assumption of nowhere zero curvature is used in its equivalent form that the second fundamental forms do not commute. These are equivalent pointwise as is seen by a straight forward calculation to establish

$$
\left\langle R^{*}(X, Y) N_{1}, N_{2}\right\rangle=\left\langle\left[A N_{1}, A N_{2}\right] X, Y\right\rangle
$$

where $[\alpha, \beta]=\alpha \beta-\beta \alpha$.

LEMMA 1. Let $\mathbf{g :} \mathbf{E}^{2} \rightarrow \mathbf{E}^{4}$ be an isometric immersion with nowhere zero normal curvature. Then there exist unique global $C^{\infty}$ unit normal vector fields $N_{1}$ and $N_{2}$ satisfying $\operatorname{det} A N_{1}=0=\operatorname{det} A N_{2}$.

Proof. The pointwise existence follows from the first Gauss equation which is equivalent to $\operatorname{det} A N+\operatorname{det} A N^{\perp}=0$, where $N$ and $N^{\perp}$ are 
orthogonal (the orthogonal operation $\perp$ requires an orientation of the normal bundle, which exists since $\mathbf{E}^{2}$ is contractible). The uniqueness comes from the noncommutivity of the second fundamental forms. The $C^{\infty}$ differentiability is demonstrated below.

The mean curvature vector field $H$ is well defined by $H=(\operatorname{tr} A N) N$ $+\left(\operatorname{tr} A N^{\perp}\right) N^{\perp}$, for arbitrary orthonormal fields $N, N^{\perp}$. It is nowhere zero as can be easily seen by using the frame $N_{1}, N_{2}$ (for otherwise, the symmetry of the second fundamental form and the condition det $A N_{1}=0$ $=\operatorname{tr} A N_{1}$ imply that $A N_{1}$ is the zero transformation, a contradiction).

Define $\sigma: \mathbf{E}^{2} \rightarrow \mathbf{E}^{1}$ by $N_{1}=\cos \sigma H_{1}+\sin \sigma H_{2}$ where $H_{1}=H /\|H\|$ and $H_{2}=H_{1}^{\perp}$. It suffices to show that $\sigma$ is $C^{\infty}$. The eigenvectors of $A H_{t}$ are $C^{\infty}$ (since the eigenvalues are distinct) and with respect to a basis of eigenvectors for, say, $\mathrm{AH}_{2}$, we can write

$$
A H_{1}=\left(\begin{array}{cc}
p & q \\
q & r
\end{array}\right), \quad A H_{2}=\left(\begin{array}{cc}
\tau & 0 \\
0 & -\tau
\end{array}\right) .
$$

The first Gauss equation, $\tau^{2}=p r-q^{2}$, implies that $0=\operatorname{det} A N_{1}=$ $\tau^{2} \cos 2 \sigma+(\tau / 2)(r-p) \sin 2 \sigma$. Hence $\sigma=\frac{1}{2} \operatorname{arccot}((p-r) / 2 \tau)$ is $C^{\infty}$. Note that $\tau \neq 0$ and $\sin 2 \sigma \neq 0$ since $A H_{2} \neq 0$.

If the normal curvature is allowed to be zero, then the normal fields $N_{1}$ and $N_{2}$ may not even be continuous. An example is given in $\S 3$.

If $N$ is any unit normal vector field for $g: \mathbf{E}^{2} \rightarrow \mathbf{E}^{4}$, then the associated normal connection 1-form is denoted by $\Theta_{N}$. The associated tangent vector field $Z_{N}$ is defined by $\Theta_{N}(\cdot)=\left\langle\cdot, Z_{N}\right\rangle$. Observe that $Z_{N}=-Z_{N^{\perp}}$. We assume throughout that the tangent and normal bundles over $\mathbf{E}^{2}$ have a preferred orientation so that the orthogonal operation $\perp$ is well-defined.

LEMMA 2. Let $\mathbf{g}: \mathbf{E}^{2} \rightarrow \mathbf{E}^{4}$ be an isometric immersion with nowhere zero normal curvature. Then $Z_{N_{1}}^{\perp}$ lies in the kernel of either $A N_{1}$ or $A N_{2}$.

Proof. We will first show that $Z_{N_{1}}^{\perp} \in \operatorname{ker}\left(A N_{2}\right)$ at $p \in \mathbf{R}^{2}$, under the assumption that $Z_{N_{1}}^{\perp} \notin \operatorname{ker}\left(A N_{1}\right)$ at $p$. Let $X_{i}, X_{i}^{\perp}$ be the eigenvectors of $A N_{i}$, with corresponding eigenvalues $\lambda_{i}$ and $0, i=1,2$. Let $\alpha$ (resp. $\beta$ ) be an integral curve of $X_{1}^{\perp}$ (resp. $Z_{N_{1}}^{\perp}$ ), through $p \in \mathbf{E}^{2}$.

We intend to construct two tangent fields $X, Y$ and one normal field $N$ along $\alpha$ and $\beta$ (and then extend these fields to a neighborhood of $p$ ). They will be used in a calculation of the Codazzi-Mainardi equation. Let $X=X_{1}^{\perp}$ and define $N$ along $\beta$ by $\left.N\right|_{\beta}=\left.N_{1}\right|_{\beta}$. Set $\left.Y\right|_{\beta}=\left.Z_{N_{1}}^{\perp}\right|_{\beta}$. Then $\left.\left(A_{X} N\right)\right|_{\beta}=0$ and so

$$
\left(\nabla_{Y} A_{X} N\right)_{p}=0 .
$$


Now choose $N$ and $Y$ along $\alpha$ so that $\|N\|=1, Y_{p}=\left(Z_{N_{1}}^{\perp}\right)_{p}$, and $A_{Y} N$ is parallel along $X$. The existence of $N$ and $Y$ is demonstrated as follows. Let $V$ be the parallel vector field along $\alpha$ with $V_{p}=\left(X_{1}\right)_{p}$. Set $N=\cos \rho N_{1}+$ $\sin \rho N_{2}$ and $V=b_{1} X_{1}+b_{2} X_{2}$ with $\rho(p)=0=b_{2}(p)$. We want $V=$ $A_{Y} N$, or equivalently,

$$
b_{1} X_{1}+b_{2} X_{2}=\lambda_{1} \cos \rho\left\langle Y, X_{1}\right\rangle X_{1}+\lambda_{2} \sin \rho\left\langle Y, X_{2}\right\rangle X_{2} .
$$

Choose an arbitrary $\tilde{Y}$ along $\alpha$ so that

$$
\begin{gathered}
\left\langle\tilde{Y}, X_{2}\right\rangle \neq 0, \\
X_{p} \cdot\left[\frac{b_{2}}{\lambda_{2}\left\langle\tilde{Y}, X_{2}\right\rangle}\right]+\left\langle X, Z_{N_{1}}\right\rangle_{p} \neq 0
\end{gathered}
$$

(this is easily seen to be possible since $\left\langle X, Z_{N_{1}}\right\rangle_{p} \neq 0$ by assumption).

Let $\rho=\arcsin \left(b_{2} / \lambda_{2}\left\langle\tilde{Y}, X_{2}\right\rangle\right)$ and obtain $\left.Y\right|_{\alpha}$ by adding to $\tilde{Y}$ a multiple of $X_{2}^{\perp}$ so that $\left\langle Y, X_{1}\right\rangle=b_{1} / \lambda_{1} \cos \rho$. This completely defines $N$ and $Y$ along $\alpha$, and we obtain

$$
\left(\nabla_{X} A_{Y} N\right)_{p}=0
$$

Finally extend $N$ and $Y$ arbitrarily to a neighborhood of $p$. Now,

$$
D_{X} N=(X \cdot \cos \rho) N_{1}+\cos \rho D_{X} N_{1}+(X \cdot \sin \rho) N_{2}+(\sin \rho) D_{X} N_{2} .
$$

Since $\rho(p)=0$,

$$
\begin{aligned}
\left(D_{X} N\right)_{p} & =(X \cdot \sin \rho)_{p} N_{2 p}+\left(D_{X} N_{1}\right)_{p} \\
& =\left[X \cdot \frac{b_{2}}{\lambda_{2}\langle\tilde{Y}, X\rangle}+\left\langle X, Z_{N_{1}}\right\rangle\right]_{p} N_{2 p} \neq 0
\end{aligned}
$$

From equations (1), (2), and (3), the Codazzi-Mainardi equation becomes

$$
-\left(A_{[X, Y]} N\right)_{p}=\left(A_{\gamma Z_{N}^{\perp}} N^{\perp}\right)_{p}
$$

where $\gamma$ is a nonzero function which depends on the lengths of and the angle between $X$ and $Y$. Both sides of this equation vanish because they are parallel to $X_{1}$ and $X_{2}$ respectively. Hence $\left(Z_{N}^{\perp}\right)_{p}$ lies in the kernel of $A N_{2}$. But $\left.N\right|_{\beta}=\left.N_{1}\right|_{\beta}$, i.e. $N$ is parallel in the normal bundle along $\beta$, and so $Z_{N}$ is perpendicular to $Z_{N_{1}}^{\perp}$ along $\beta$. Thus $Z_{N_{1}}^{\perp}$ is in the kernel of $A N_{2}$ at $p \in \mathbf{E}^{2}$.

It remains to show that $Z_{N_{1}}^{\perp}$ cannot be zero along a curve so that, on opposite sides of this zero curve, $Z_{N_{1}}^{\perp}$ lies in $\operatorname{ker}\left(A N_{1}\right)$ and $\operatorname{ker}\left(A N_{2}\right)$ respectively. We have already shown that $Z_{N_{1}}^{\perp}=k_{1} X_{1}^{\perp}+k_{2} X_{2}^{\perp}$ where 
$k_{1}, k_{2}$ are $C^{\infty}$ functions satisfying $k_{1} \cdot k_{2}=0$. If $k_{1}$ and $k_{2}$ vanish on opposite sides of a curve, then their derivatives vanish on the curve. But this means that the normal curvature has a zero, contradiction.

Q.E.D.

LEMMA 3. Let $g: \mathbf{E}^{2} \rightarrow \mathbf{E}^{4}$ be an isometric immersion with nowhere zero normal curvature. Then $g$ factors as the composition of isometric immersions $g=g_{1} \circ g_{2}: \mathbf{E}^{2} \rightarrow \mathbf{E}^{3} \rightarrow \mathbf{E}^{4}$.

Proof. Let $N_{1}$ and $N_{2}$ be normals in the previous lemmas with $Z_{N_{1}}^{\perp}$ in ker $A N_{2}$. Let $M$ be the subbundle of the normal bundle $\nu(g)$ generated by $N_{1}$ with the induced Riemannian data from $\nu(g)$. It is trivial to see that the Gauss and Codazzi-Mainardi equations are satisfied by $M$. From the existence theorem, $M$ is the normal bundle to an isometric immersion $g_{2}$ : $\mathbf{E}^{2} \rightarrow \mathbf{E}^{3}$.

The mapping $g_{1}$ essentially identifies $\mathbf{E}^{3}$ with the normal (sub)bundle $M$. To be more precise, first recall that a focal point is, by definition, a singularity of the identification of $\mathbf{E}^{3}$ with $M$. The nonfocal points form an open dense subset $G \subset \mathbf{E}^{3}$ on which this identification is, locally, an isometric diffeomorphism. Regarding $M$ as a subset of $\mathbf{E}^{4}$, we obtain an isometric immersion from $G$ to $\mathbf{E}^{4}$ which extends, by continuity, to all of $\mathbf{E}^{3}$. It is denoted by $g_{1}$.

Q.E.D.

So far, our arguments have been local in nature and simple connectivity has been the only topological assumption needed to apply the existence theorem. We now state a general result.

THEOREM 2. Let $U$ be an open 2-dimensional flat manifold and consider an isometric immersion $g: U \rightarrow \mathbf{E}^{4}$ with nowhere zero normal curvature. Then there exists an open 3-dimensional flat manifold $N$ and two isometric immersions $g_{1}: U \rightarrow N$ and $g_{2}: N \rightarrow \mathbf{E}^{4}$ so that $g=g_{2} \circ g_{1}$.

The manifold $N$ may be chosen as a tubular neighborhood of zero section of an appropriate line subbundle of $\nu(g)$. If $U$ (and hence $N$ ) is simply connected, then both may be regarded as open subsets of Euclidean space. Simple connectivity is not needed in Theorem 2 because the desired mappings already exist-our main effort has been to find a subbundle of $\nu(g)$ which is flat, viewed as a submanifold of $\mathbf{E}^{4}$.

Proof of Theorem 1. Hartman [1] showed that any isometric immersion $f: \mathbf{E}^{n} \rightarrow \mathbf{E}^{n+2}, n \geq 2$, (no assumption on the normal curvature) 
factors as a Riemannian product $f=g \times$ id: $\mathbf{E}^{2} \rightarrow \mathbf{E}^{n-2} \rightarrow \mathbf{E}^{4} \times \mathbf{E}^{n-2}$. If $f$ has nonzero normal curvature, then so does $g$. By Lemma $3, g$ factors as a composition, and hence so does $f$.

Q.E.D.

3. Counterexamples. If the normal curvature is allowed to vanish, then $f: \mathbf{E}^{n} \mapsto \mathbf{E}^{n+2}$ may not factor as a composition. The idea behind the counterexample is to roll $\mathbf{E}^{2}$, regarded as complementary half-planes $P_{1}$ and $P_{2}$, into two distinct hyperplanes in $\mathbf{E}^{4}$. To insure that the two rollings fit together smoothly, we may assume that near the boundary of each half-plane, the rolling "flattens out" (like $x \rightarrow e^{-1 / x^{2}}$ near 0 ). In this way, we obtain unique normals $N_{1}$ and $N_{2}$ on each open half-plane $P_{i}$. But these do not extend continuously across their common boundary for they are uniquely determined on each half-plane by rollings into different hyperplanes.

\section{REFERENCES}

[1] P. Hartman, On the isometric immersions in Euclidean space of manifolds with nonnegative sectional curvature II, Trans. Amer. Math. Soc., 147 (1970), 529-540.

[2] P. Hartman and L. Nirenberg, On spherical image maps whose Jacobians do not change signs, Amer. J. Math., 81 (1959), 901-920.

[3] W. S. Massey, Surfaces of Gaussian curvature zero in Euclidean space, Tohoku Math. J., 14 (1962), 73-79.

[4] A. V. Pogorelov, Extensions of the theorem of Gauss on spherical representations to the case of surfaces of bounded extrinsic curvature, Dokl. Akad. Nauk SSSR (N.S.), 111 (1956), 945-947.

[5] J. J. Stoker, Developable surfaces in the large, Comm. Pure and Applied Math., XIV, no. 3, (1961), 627-635.

[6] R. Szczarba, On existence and rigidity of isometric immersions, Bull. Amer. Math. Soc., 75 (1969), 783-787.

[7] L. Whitt, Isometric homotopy and codimension two isometric immersions of the $n$-sphere into Euclidean space, J. Differential Geom., 14 (1979), 295-302.

Received February 17, 1982 and in revised form January 22, 1985.

\section{INRI}

Building Two, SuITE A

710 DeNBigh BlVD.

NEWPORT NEWS, VA 23602 



\section{PACIFIC JOURNAL OF MATHEMATICS EDITORS}

\author{
V. S. VARADARAJAN (Managing Editor) \\ University of California \\ Los Angeles, CA 90024 \\ Charles R. DePrima \\ California Institute of Technology \\ Pasadena, CA 91125 \\ R. FInN \\ Stanford University \\ Stanford, CA 94305
}

\section{HeRmanN FlaschKa \\ University of Arizona \\ Tucson, AZ 85721}

Ramesh A. Gangolli

University of Washington

Seattle, WA 98195

ROBION KIRBY

University of California

Berkeley, CA 94720

\section{C. MOORE}

University of California

Berkeley, CA 94720

\section{H. SAMELSON}

Stanford University

Stanford, CA 94305

HAROLD STARK

University of California, San Diego

La Jolla, CA 92093

\section{ASSOCIATE EDITORS}
R. ARENS
E. F. BECKENBACH
B. H. NeumanN
F. Wolf
K. Yoshida (1906-1982)

\section{SUPPORTING INSTITUTIONS}
CALIFORNIA INSTITUTE OF TECHNOLOGY

\author{
UNIVERSITY OF SOUTHERN CALIFORNIA \\ STANFORD UNIVERSITY \\ UNIVERSITY OF HAWAII \\ UNIVERSITY OF TOKYO \\ UNIVERSITY OF UTAH \\ WASHINGTON STATE UNIVERSITY \\ UNIVERSITY OF WASHINGTON
}
UNIVERSITY OF OREGON

\begin{abstract}
UNIVERSITY OF ARIZONA
UNIVERSITY OF BRITISH COLUMBIA

UNIVERSITY OF CALIFORNIA

MONTANA STATE UNIVERSITY

UNIVERSITY OF NEVADA, RENO

NEW MEXICO STATE UNIVERSITY

OREGON STATE UNIVERSITY
\end{abstract}

The Supporting Institutions listed above contribute to the cost of publication of this Journal, but they are not owners or publishers and have no responsibility for its content or policies.

Mathematical papers intended for publication in the Pacific Journal of Mathematics should be in typed form or offset-reproduced (not dittoed), double spaced with large margins. Please do not use built up fractions in the text of the manuscript. However, you may use them in the displayed equations. Underline Greek letters in red, German in green, and script in blue. The first paragraph must be capable of being used separately as a synopsis of the entire paper. In particular it should contain no bibliographic references. Please propose a heading for the odd numbered pages of less than 35 characters. Manuscripts, in triplicate, may be sent to any one of the editors. Please classify according to the scheme of Math. Reviews, Index to Vol. 39. Supply name and address of author to whom proofs should be sent. All other communications should be addressed to the managing editor, or Elaine Barth, University of California, Los Angeles, California 90024.

There are page-charges associated with articles appearing in the Pacific Journal of Mathematics. These charges are expected to be paid by the author's University, Government Agency or Company. If the author or authors do not have access to such Institutional support these charges are waived. Single authors will receive 50 free reprints; joint authors will receive a total of 100 free reprints. Additional copies may be obtained at cost in multiples of 50 .

The Pacific Journal of Mathematics is issued monthly as of January 1966. Regular subscription rate: $\$ 190.00$ a year (5 Vols., 10 issues). Special rate: $\$ 66.00$ a year to individual members of supporting institutions.

Subscriptions, orders for numbers issued in the last three calendar years, and changes of address should be sent to Pacific Journal of Mathematics, P.O. Box 969, Carmel Valley, CA 93924, U.S.A. Old back numbers obtainable from Kraus Periodicals Co., Route 100, Millwood, NY 10546.

The Pacific Journal of Mathematics at P.O. Box 969, Carmel Valley, CA 93924 (ISSN 0030-8730) publishes 5 volumes per year. Application to mail at Second-class postage rates is pending at Carmel Valley, California, and additional mailing offices. Postmaster: Send address changes to Pacific Journal of Mathematics, P.O. Box 969, Carmel Valley, CA 93924.

PUBLISHED BY PACIFIC JOURNAL OF MATHEMATICS, A NON-PROFIT CORPORATION

Copyright $\odot 1985$ by Pacific Journal of Mathematics 


\section{Pacific Journal of Mathematics}

Vol. 119, No. $2 \quad$ June, 1985

Mustafa Agah Akcoglu and Meira Falkowitz (Soshniak), A general local

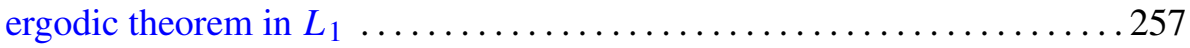

W. Wistar (William) Comfort and Lewis Chandlee Robertson,

Cardinality constraints for pseudocompact and for totally dense

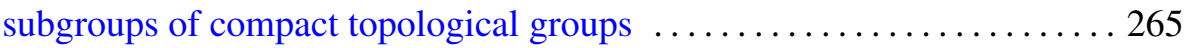

John Morse Delaurentis and Boris G. Pittel, Random permutations and

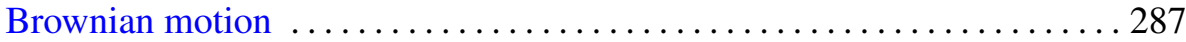

José Esteban Galé, Gel'fand theory in algebras of differentiable functions on Banach spaces ......................................... 303

Harry Gingold, On the location of zeroes of oscillatory solutions of

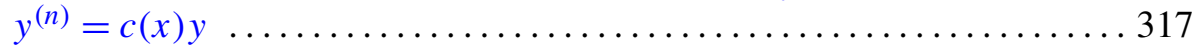

Kei Ji Izuchi, Zero sets of interpolating Blaschke products .............337

Mahesh Nerurkar, Ergodic continuous skew product actions of amenable

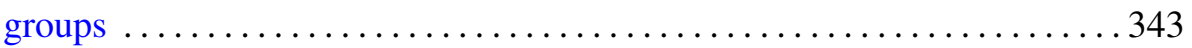

R. Owens, A maximal function characterization of a class of Hardy

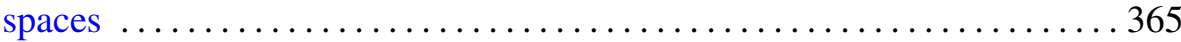

Judith Anne Packer, Point spectrum of ergodic abelian group actions and the corresponding group-measure factors $\ldots \ldots \ldots \ldots \ldots \ldots \ldots \ldots \ldots \ldots \ldots \ldots$

Judith Anne Packer, On the embedding of subalgebras corresponding to quotient actions in group-measure factors $\ldots \ldots \ldots \ldots \ldots \ldots \ldots \ldots . \ldots 4$

Iain Raeburn and Joseph L. Taylor, The bigger Brauer group and étale cohomology

David Rosen, The Diophantine equation $a x+b y=c$ in $Q(\sqrt{5})$ and other number fields

Mau-Hsiang Shih and Kok Keong Tan, Noncompact sets with convex sections

Lee Barlow Whitt, Codimension two isometric immersions between Euclidean spaces

Rodney Ian Yager, Iwasawa theory for the anticyclotomic extension 